IOS Press

\title{
Effect of postharvest handling practices on phytochemical concentrations and bioactive potential in wild blueberry fruit
}

\author{
Sally J. Gustafson ${ }^{\mathrm{a}}$, Gad G. Yousef ${ }^{\mathrm{b}}$, Michael A. Grusak ${ }^{\mathrm{c}}$ and Mary Ann Lila ${ }^{\mathrm{b}, *}$ \\ ${ }^{a}$ USDA-ARS at North Carolina Research Campus, Kannapolis, NC, USA \\ ${ }^{\mathrm{b}}$ Plants for Human Health Institute, North Carolina State University, Kannapolis, NC, USA \\ ${ }^{\mathrm{c}}$ USDA-ARS Children's Nutrition Research Center, Department of Pediatrics, Baylor College of Medicine, \\ Houston, TX, USA
}

Received 10 February 2012; accepted 8 May 2012

\begin{abstract}
In this study, we quantified anthocyanin (ANC), proanthocyanidin (PAC), and chlorogenic acid (CA) concentrations in wild blueberry fruit (WBB) exposed to a variety of postharvest handling practices relevant to consumers and to industry. Additionally, we analyzed the bioactive potential of WBB subjected to common culinary preparations such as baking, boiling, and microwaving. Levels of ANC, PAC, and CA in individually quick frozen (IQF) WBB that had been subjected to temperature fluctuations, which are often encountered during distribution and handling for retail sales, dropped by approximately 8,43 , and $60 \%$, respectively, compared to an IQF WBB composite that was stored continuously from harvest at $-80^{\circ} \mathrm{C}$. Baking IQF WBB reduced ANC, PAC, and CA concentrations by 11.2, 14.6, and 10.6\%, respectively, and boiling decreased ANC, PAC, and CA concentrations by a minimum of 7.4, 14.4, and 36.8\%, respectively. Microwaving IQF WBB for 1 minute increased ANC concentrations by $12.9 \%$ but exposure to 3 and 5 minutes resulted in significant decreases ( 29.8 and $81.6 \%$, respectively). PAC concentrations in IQF WBB exposed to microwaves for 1, 3, and 5 minutes decreased by $14.3,5.4$, and $87.1 \%$, respectively, whereas CA concentrations were not significantly impacted. At a concentration of $25 \mu \mathrm{g} / \mathrm{ml}$, baked and boiled IQF WBB extracts maintained the ability to inhibit lipopolysaccharide (LPS)-induced reactive oxygen species (ROS) in SH-SY5Y cells. Extracts of WBB that were microwaved for 1 or 3 minutes retained bioactivity in our model for inflammation, while those microwaved for 5 minutes were unable to inhibit LPS-induced ROS.
\end{abstract}

Keywords: Blueberry, polyphenols, reactive oxygen species, HPLC, anthocyanins, polyphenol oxidase

\section{Introduction}

Blueberry fruit has been reported to decrease cardiovascular risk factors, enhance insulin sensitivity, improve cognitive abilities, and decrease oxidative stress in the central nervous system [1-4]. Additionally, extracts of blueberries have been shown to exhibit a bioactive potential capable of inhibiting cancer cell proliferation [5]. Wild blueberry (WBB) fruit, in particular, has a complex and unique polyphenolic phytochemical profile that boasts antioxidant and anti-inflammatory potential. Postharvest handling parameters have potential to impact the bioactive polyphenolic concentrations in WBB available to consumers.

According to the Mintel Global New Products Database, 1,200 new blueberry products were introduced in 2010 [6]. The North American Blueberry Council reported that frozen blueberry consumption increased from 15 ounces per person in 2008 to 16 ounces per person in 2009 [7]. These trends suggest that consumers and industry are familiar

*Corresponding author: Dr. Mary Ann Lila, Director, Plants for Human Health Institute, North Carolina State University, 600 Laureate Way, Kannapolis, NC 28081, USA. Tel.: +1 704250 5407; Fax: +1 704250 5409; E-mail: mlila@ ncsu.edu. 
with recent blueberry research findings and that individuals are increasingly interested in incorporating blueberries into their diets, due to their potential health benefits. Blueberries contain high concentrations of anthocyanins and more specifically, it has been established that wild blueberries (WBB) have even higher levels of anthocyanins, total phenolics, and ORAC antioxidant capacities compared to other blueberry varieties [8, 9]. Wild blueberries are commercially available at grocery markets as individually quick frozen (IQF) berries, and are the most common blueberry type used in cereals, yogurts, muffins and other processed foods available to consumers [9].

Despite the increased consumption of this berry fruit, there remains limited information with regard to how postharvest processing, storage, and culinary preparations influence polyphenolic levels and health benefits in blueberries available to consumers. To better understand the effects of postharvest handling on wild blueberries, we measured anthocyanin, proanthocyanidin and chlorogenic acid concentrations following a series of postharvest handling techniques that are relevant to consumers and to the berry industry. Lastly, we investigated polyphenolic variations associated with common culinary preparations and how these changes influenced the bioactivity of WBB in a cell culture model for inflammation.

\section{Material and methodology}

\subsection{Chemicals}

Cyanidin-3-O-glucoside and procyanidin A2 were purchased from Chroma Dex (Irvine, CA, USA). SH-SY5Y cells, Dulbecco's modified eagle medium nutrient mixture F-12 HAM (DMEM:F-12), and fetal bovine serum were purchased from ATCC (Manassas, VA, USA). 2',7'-Dichlorofluorescindiacetate (DCF-DA), L-glutathione, penicillinstreptomycin, trypsin-EDTA, trifluoroacetic acid (TFA), and chlorogenic acid (CA) were all purchased from Sigma (St. Louis, MO, USA). Other supplies were purchased from Fisher Scientific (Atlanta, GA, USA).

\subsection{Berry source}

IQF low bush wild blueberries (Vaccinium angustifolium Aiton) were obtained from the Wild Blueberry Association of North America (WBANA) (Old Town, ME, USA). The WBB fruit was a composite of fruits from all major growing sites including Prince Edward Island, New Brunswick, Nova Scotia, and Maine. The IQF composite was made in the fall of 2010 and stored at $-80^{\circ} \mathrm{C}$ in our laboratory freezer until use. Freeze dried (FD) wild blueberry material was prepared by Future Ceuticals (Momence, IL, USA) using blueberries from the same source. Packaged IQF low bush wild blueberries (Dole Food Company, Atwater, CA, USA) that were held at $-20^{\circ} \mathrm{C}$, for no longer than 12 months, before distribution to retail grocery markets were acquired and stored at $-80^{\circ} \mathrm{C}$ in our laboratory freezer until analysis.

\subsection{Processing, storage, and culinary preparation of wild blueberries}

IQF wild blueberry composite stored continually at $-80^{\circ} \mathrm{C}$ from the time of harvest, FD wild blueberry powder, and IQF wild blueberries obtained from a supermarket source were all analyzed to determine how postharvest processing techniques affect polyphenolic concentrations. Additionally, we subjected IQF $-80^{\circ} \mathrm{C}$ berries to storage conditions relevant to consumers including $4^{\circ} \mathrm{C}$ and room temperature $\left(23^{\circ} \mathrm{C}\right)$ for up to 10 days, and to two freeze-thaw cycles $\left(-80^{\circ} \mathrm{C}\right.$ to $23^{\circ} \mathrm{C}, 24 \mathrm{hrs}$ at each temperature) followed by a final freezing step. Lastly, $\mathrm{IQF}-80^{\circ} \mathrm{C}$ were subjected to culinary preparations including baking, boiling and microwaving. Berries taken directly from $-80^{\circ} \mathrm{C}$ storage were baked in $250 \mathrm{ml}$ glass beakers at $175^{\circ} \mathrm{C}\left(350^{\circ} \mathrm{F}\right)$ and $220^{\circ} \mathrm{C}\left(425^{\circ} \mathrm{F}\right)$ for up to one hour, placed in $200 \mathrm{ml}$ boiling water $\left(200^{\circ} \mathrm{C}\right.$ ) for up to 10 minutes, or microwaved (Avanti, $0.9 \mathrm{Cu}$. Ft, 115 Volts $\mathrm{AC}, 60 \mathrm{~Hz}, 900$ Watts) for up to 5 minutes. Upon completion of each treatment, samples were extracted and prepared for analyses. Untreated or stored IQF WBB were extracted using $1 \%$ acetic acid in $\mathrm{MeOH}$ whereas FD WBB and WBB subjected to culinary preparations were extracted using $1 \%$ acetic acid in $\mathrm{H}_{2} \mathrm{O} / \mathrm{MeOH}(80: 20)$ to account for water loss during postharvest procedures. Berry samples were extracted three times; briefly, berries were blended (first extraction, $30 \mathrm{ml}$ ) or the pellets were mixed (subsequent extractions, $20 \mathrm{ml}$ ) in extraction buffer, the samples were then centrifuged ( $4000 \mathrm{rpm}$ ) at $20^{\circ} \mathrm{C}$ for $20 \mathrm{~min}$, 
the supernatants were collected, and a final volume $(100 \mathrm{ml})$ was achieved by addition of extraction buffer. Initial weight of each berry sample was recorded (approximately $20 \mathrm{~g}$ ) prior to experimentation and was used to calculate the concentrations of polyphenolic compounds per gram of berry weight for each sample. Additional consideration was given to the FD wild blueberry powder and the weight of our samples were scaled back to take into consideration the $80 \%$ weight decrease in blueberry material due to water loss that occurs during the freeze drying process. Three separate experiments were run for each condition.

\subsection{HPLC analysis}

HPLC analyses were conducted using a 1260 HPLC with a diode array detector (Agilent Technologies, Santa Clara, CA, USA). Samples were prepared by filtering through $0.45 \mu$ m nylon filters (Fisher Scientific, GA, USA) before injection. Separation was performed with a reversed-phase Supelcosil-LC-18 column $(25 \mathrm{~mm} \times 4.6 \mathrm{~mm} \times 5 \mu \mathrm{m})$ (Sigma, St. Louis, MO, USA) according to previous methods [10, 11]. Chemstation software (Agilent Technologies Inc.) was used as a system controller and for data processing. For anthocyanin (ANC) separation, the mobile phase consisted of $5 \%$ formic acid in $\mathrm{H}_{2} \mathrm{O}(\mathrm{A})$ and $100 \%$ methanol (B); cyanidin-3-O-glucoside (1.0, 0.5. 0.25 and $0.0625 \mathrm{mg} / \mathrm{ml}$ ) was used as an external standard, and the 17 major ANC peaks were detected at $520 \mathrm{~nm}$. Using commercial standards, LC-MS/MS, and published data about anthocyainins in wild blueberries, individual ANC peaks were previously identified [11]. The mobile phase for chlorogenic acid consisted of $94.9 \% \mathrm{H}_{2} \mathrm{O}, 5 \%$ acetonitrile, and $0.1 \%$ formic acid (A) and $94.9 \%$ acetonitrile, $5 \% \mathrm{H}_{2} \mathrm{O}$, and $0.1 \%$ formic acid (B); chlorogenic acid (1.0, 0.5, and $0.25 \mathrm{mg} / \mathrm{ml}$ ) was used as an external standard and detection was recorded at $280 \mathrm{~nm}$. Concentrations were calculated and presented as equivalents of corresponding external standards per gram of fresh weight. WBB samples were analyzed in triplicates.

\subsection{Total anthocyanins and proanthocyanidins}

Total monomeric anthocyanins were estimated by a $\mathrm{pH}$ differential method using a UV-2450 Shimadzu spectrophotometer (Portland, OR, USA) and $1 \mathrm{~cm}$ path length cuvette [12]. Absorbance was measured at 510 and $700 \mathrm{~nm}$ in buffers at $\mathrm{pH} 1.0$ and 4.5, using $\mathrm{A}=\left(\mathrm{A}_{510}-\mathrm{A}_{700}\right) \mathrm{pH}_{1.0}-\left(\mathrm{A}_{510}-\mathrm{A}_{700}\right) \mathrm{pH}_{4.5}$. Anthocyanin concentration was calculated as milligrams of cyanidin-3- $O$-glucoside equivalent per gram fresh weight, using a molar extinction coefficient of 26,900 and molecular weight of 449. Total proanthocyanidins were determined with a 4dimethylaminocinnamaldehyde (DMAC) spectrophotometric assay as previously described [13]; absorbance was measured using a SpectraMax M3 multi-mode microplate reader (Sunnyvale, CA, USA). Results were expressed as milligrams of procyanidin A2 equivalent per gram of fresh weight.

\subsection{Quantification of reactive oxygen species production}

SH-SY5Y human neuroblastoma cells were cultured in a humidified atmosphere $\left(5 \% \mathrm{CO}_{2}, 37^{\circ} \mathrm{C}\right)$ and grown in DMEM: F-12 medium supplemented with $10 \%$ fetal bovine serum, $100 \mathrm{U} / \mathrm{ml}$ Penicillin, and $100 \mathrm{U} / \mathrm{ml}$ Streptomycin in $100 \mathrm{~mm}$ dishes (Greiner Bio-one, Monroe, NC, USA). Cultures were replenished with fresh medium every 48 to 72 hours. Cells were harvested with trypsin $(0.5 \mathrm{mg} / \mathrm{ml}) /$ EDTA $(0.2 \mathrm{mg} / \mathrm{ml})$ in phosphate buffered saline (PBS), centrifuged for $2 \mathrm{~min}$ at $125 \times \mathrm{g}$, suspended in new medium, and plated to tissue culture-treated 6 well plates (Greiner Bio-one, Monroe, NC, USA) for experimentation. Cell cultures at passage 8 were maintained for 72 hours and were then serum starved overnight prior to treatment. For this set of experiments, stock solutions were prepared by freeze drying the WBB extracts and then reconstituting the dry material in 70:30 methanol-water. Stock WBB extracts were diluted immediately prior to use in DMEM at a final assay concentration of $25 \mu \mathrm{g} / \mathrm{ml}$. Cultures were incubated (1 hr) with $1 \mathrm{mM}$ glutathione $(\mathrm{GSH})$ or $25 \mu \mathrm{g} / \mathrm{ml}$ blueberry extract in conjunction with DCF-DA (10 $\mu \mathrm{M})$. Cultures were then exposed to $1 \mu \mathrm{g} / \mathrm{ml}$ lipopolysaccharide (LPS) for 1 hour - during which DCF-DA is oxidized to DCF by $\mathrm{H}_{2} \mathrm{O}_{2}$. Cells were washed and harvested in PBS and $200 \mu \mathrm{l}$ of sample was transferred to a black 96 well plate (Corning) for analysis. ROS formation was quantified as maximum DCF-Fluorescence intensity using a SpectraMax M3 multi-mode microplate reader (Sunnyvale, CA, USA) (485 nm excitation, 538 emission). A $10 \mu l$ aliquot of each 
sample was used for protein quantification with a BCA protein assay kit according to manufacturer's instructions (Thermo Scientific, Rockford, IL, USA). All values were normalized and reported as fluorescence intensity per $\mu \mathrm{g}$ of protein.

\subsection{Statistical analysis}

All results are expressed as means \pm SD of three separate experiments unless otherwise stated. The statistical evaluation of the results was performed by one-way analysis of variance (ANOVA) followed by Bonferroni's Multiple Comparison Test using GraphPad Prism 5 (GraphPad Software, San Diego, CA, USA). Significance was defined as $p<0.05$ and significant changes are indicated as $* p<0.05$.

\section{Results}

\subsection{Postharvest processing and storage influence anthocyanin concentration in IQF wild blueberries}

ANC concentrations were determined in IQF WBB stored continuously at $-80^{\circ} \mathrm{C}$, FD, or stored at $-20^{\circ} \mathrm{C}$ during retail handling (Table 1). Results reveal that IQF WBB stored at $-80^{\circ} \mathrm{C}$ or FD retain similar total ANC concentrations $(\mathrm{mg} / \mathrm{g})(2.66 \pm 0.015$ and $2.64 \pm 0.007$, respectively). Of the 17 ANC detected (Fig. 1), 5 specific compounds (cyanidin-3-galactoside, cyanidin-3-glucoside, petunidin-3-glucoside, peonidin-3-galactoside, and malvidin-3-galactoside) in the IQF WBB obtained from a supermarket source were significantly lower than those stored continuously at $-80^{\circ} \mathrm{C}$. Results indicated that total ANC in IQF $\left(-20^{\circ} \mathrm{C}\right)$ deviated from control berries by $8.345 \%$. These data suggest that temperature fluctuations associated with the transportation and distribution of WBB and storage in retail stores may result in some thermal degradation of ANC prior to consumer purchase.

Next, IQF WBB $\left(-80^{\circ} \mathrm{C}\right)$ were subjected to three additional storage conditions in an attempt to determine if storage conditions relevant to consumer practices influenced ANC concentrations (Table 1). IQF WBB stored at all three conditions (refrigerated storage, room temperature, or subjected to a freeze-thaw cycle) showed significant decreases in many of the specific ANC concentrations measured. These results agree with previous reports that indicate ANC concentrations decrease in fruit or fruit products that are stored [14-16]. Furthermore, IQF berries stored at $4^{\circ} \mathrm{C}$ and room temperature $\left(23^{\circ} \mathrm{C}\right)$ for up to 10 days showed significant decreases in total ANC concentrations over time (Table 2).



Fig. 1. Anthocyanin peak identification acquired at $520 \mathrm{~nm}$ using HPLC analysis in IQF wild blueberries. Peak numbers correspond to: 1. delphinidin-3-galactoside, 2. delphinidin-3-glucoside, 3. cyanidin-3-galactoside, 4. delphinidin-3-arabinoside, 5. cyanidin-3-glucoside, 6. cyanidin-3-arabinoside, 7. petunidin-3-glucoside, 8. peonidin-3-galactoside, 9. peonidin-3-arabinoside, 10. malvidin-3-galactoside, 11. malvidin3-glucoside, 12. malvidin-3-arabinoside, 13. delphinidin-6-acetyl-3-glucoside, 14. cyanidin-6-acetyl-3-glucoside, 15. malvidin-6-acetyl-3galactoside, 16. petunidin-6-acetyl-3-glucoside, 17. malvidin-6-acetyl-3-glucoside. HPLC method and analysis are described in the material and methodology section. 
Table 1

Anthocyanin concentrations in individually quick-frozen wild blueberries following postharvest processing and storage

\begin{tabular}{|c|c|c|c|c|c|c|}
\hline \multirow[b]{2}{*}{ Peak } & \multicolumn{5}{|c|}{ Anthocyanins (mg/g) (Processing and storage) } & \multirow[b]{2}{*}{ Freeze-thaw } \\
\hline & $\mathrm{IQF}\left(-80^{\circ} \mathrm{C}\right)$ & $\mathrm{IQF}(\mathrm{FD})$ & $\mathrm{IQF}\left(-20^{\circ} \mathrm{C}\right)$ & $4^{\circ} \mathrm{C}$ (5 days) & $23^{\circ} \mathrm{C}$ (5 days) & \\
\hline 1 & $0.245 \pm 0.036$ & $0.243 \pm 0.005$ & $0.201 \pm 0.012$ & $0.135 \pm 0.010^{*}$ & $0.100 \pm 0.003^{*}$ & $0.095 \pm 0.019^{*}$ \\
\hline 2 & $0.262 \pm 0.007$ & $0.269 \pm 0.006$ & $0.238 \pm 0.029$ & $0.146 \pm 0.013^{*}$ & $0.154 \pm 0.004^{*}$ & $0.105 \pm 0.011^{*}$ \\
\hline 3 & $0.120 \pm 0.018$ & $0.110 \pm 0.002$ & $0.087 \pm 0.005^{*}$ & $0.081 \pm 0.008^{*}$ & $0.050 \pm 0.002 *$ & $0.058 \pm 0.009 *$ \\
\hline 4 & $0.161 \pm 0.025$ & $0.162 \pm 0.004$ & $0.149 \pm 0.011$ & $0.095 \pm 0.002 *$ & $0.080 \pm 0.002 *$ & $0.066 \pm 0.007 *$ \\
\hline 5 & $0.121 \pm 0.005$ & $0.117 \pm 0.003$ & $0.102 \pm 0.005^{*}$ & $0.095 \pm 0.011^{*}$ & $0.090 \pm 0.005^{*}$ & $0.077 \pm 0.006^{*}$ \\
\hline 6 & $0.143 \pm 0.013$ & $0.133 \pm 0.002$ & $0.114 \pm 0.007$ & $0.086 \pm 0.006^{*}$ & $0.071 \pm 0.003^{*}$ & $0.063 \pm 0.012^{*}$ \\
\hline 7 & $0.230 \pm 0.001$ & $0.204 \pm 0.004$ & $0.190 \pm 0.023^{*}$ & $0.136 \pm 0.011^{*}$ & $0.127 \pm 0.004 *$ & $0.099 \pm 0.009^{*}$ \\
\hline 8 & $0.046 \pm 0.005$ & $0.045 \pm 0.000$ & $0.036 \pm 0.003^{*}$ & $0.035 \pm 0.002 *$ & $0.026 \pm 0.002 *$ & $0.029 \pm 0.003^{*}$ \\
\hline 9 & $0.094 \pm 0.009$ & $0.086 \pm 0.001$ & $0.082 \pm 0.007$ & $0.058 \pm 0.003^{*}$ & $0.102 \pm 0.005$ & $0.044 \pm 0.005^{*}$ \\
\hline 10 & $0.325 \pm 0.012$ & $0.321 \pm 0.005$ & $0.278 \pm 0.016^{*}$ & $0.247 \pm 0.014^{*}$ & $0.154 \pm 0.004 *$ & $0.203 \pm 0.028^{*}$ \\
\hline 11 & $0.333 \pm 0.007$ & $0.357 \pm 0.006$ & $0.354 \pm 0.036$ & $0.280 \pm 0.022 *$ & $0.235 \pm 0.008^{*}$ & $0.222 \pm 0.023^{*}$ \\
\hline 12 & $0.138 \pm 0.013$ & $0.144 \pm 0.002$ & $0.143 \pm 0.014$ & $0.112 \pm 0.010$ & $0.110 \pm 0.007 *$ & $0.091 \pm 0.013^{*}$ \\
\hline 13 & $0.091 \pm 0.010$ & $0.092 \pm 0.002$ & $0.090 \pm 0.014$ & $0.044 \pm 0.012 *$ & $0.057 \pm 0.003^{*}$ & $0.028 \pm 0.005^{*}$ \\
\hline 14 & $0.042 \pm 0.001$ & $0.053 \pm 0.001$ & $0.051 \pm 0.009$ & $0.046 \pm 0.007$ & $0.046 \pm 0.000$ & $0.036 \pm 0.003$ \\
\hline 15 & $0.087 \pm 0.014$ & $0.069 \pm 0.017$ & $0.077 \pm 0.002$ & $0.060 \pm 0.007 *$ & $0.045 \pm 0.000^{*}$ & $0.047 \pm 0.005^{*}$ \\
\hline 16 & $0.060 \pm 0.006$ & $0.061 \pm 0.016$ & $0.066 \pm 0.012$ & $0.042 \pm 0.010$ & $0.045 \pm 0.000$ & $0.031 \pm 0.002 *$ \\
\hline 17 & $0.162 \pm 0.020$ & $0.174 \pm 0.003$ & $0.179 \pm 0.039$ & $0.150 \pm 0.025$ & $0.129 \pm 0.002$ & $0.105 \pm 0.015^{*}$ \\
\hline Total & $2.66 \pm 0.015$ & $2.641 \pm 0.007$ & $2.438 \pm 0.018$ & $1.846 \pm 0.012$ & $1.619 \pm 0.004$ & $1.399 \pm 0.013$ \\
\hline
\end{tabular}

Berries were obtained from three sources post-processing, extracted and analyzed using HPLC as described in the methods section. Peak numbers correspond to individual anthocyanins denoted in Fig. 1. Additionally, IQF WBB $\left(-80^{\circ} \mathrm{C}\right)$ were subjected to three storage conditions, extracted and analyzed. *Represents $p<0.05$ compared to control, IQF $\left(-80^{\circ} \mathrm{C}\right)$. All data represent the mean of at least 3 independent experiments $\pm \mathrm{SD}$.

Table 2

Total anthocyanin concentrations and percent decreases in individually quick-frozen wild blueberries following postharvest storage

\begin{tabular}{|c|c|c|c|c|}
\hline \multirow[b]{2}{*}{ Days } & \multicolumn{4}{|c|}{ Total anthocyanins (mg/g) (Postharvest storage for 10 days) } \\
\hline & $4^{\circ} \mathrm{C}$ & $\%$ decrease at $4^{\circ} \mathrm{C}$ & $23^{\circ} \mathrm{C}$ & $\%$ decrease at $23^{\circ} \mathrm{C}$ \\
\hline 0 & $2.660 \pm 0.015$ & 0 & $2.660 \pm 0.015$ & 0 \\
\hline 1 & $2.327 \pm 0.011^{*}$ & 12.52 & $1.930 \pm 0.010^{*}$ & 27.46 \\
\hline 2 & $1.987 \pm 0.001 *$ & 25.31 & $1.714 \pm 0.007 *$ & 35.55 \\
\hline 3 & $2.001 \pm 0.001 *$ & 24.76 & $1.628 \pm 0.006^{*}$ & 38.81 \\
\hline 4 & $1.753 \pm 0.005^{*}$ & 34.08 & $1.226 \pm 0.010^{*}$ & 53.91 \\
\hline 5 & $1.846 \pm 0.012 *$ & 30.57 & $1.619 \pm 0.004 *$ & 39.17 \\
\hline 6 & $1.846 \pm 0.001^{*}$ & 30.62 & $1.439 \pm 0.005^{*}$ & 45.91 \\
\hline 7 & $1.573 \pm 0.008^{*}$ & 40.85 & $1.492 \pm 0.006^{*}$ & 43.92 \\
\hline 8 & $1.604 \pm 0.001^{*}$ & 39.69 & $1.401 \pm 0.005^{*}$ & 47.31 \\
\hline 9 & $1.701 \pm 0.002 *$ & 36.04 & $1.464 \pm 0.005^{*}$ & 44.95 \\
\hline 10 & $1.485 \pm 0.002 *$ & 42.52 & $1.565 \pm 0.006^{*}$ & 41.16 \\
\hline
\end{tabular}

IQF berries were stored at indicated temperatures without exposure to light prior to extraction and analysis using HPLC as described in the methods section. Days indicate how many days the IQF blueberry samples were stored. $*$ Represents $p<0.05$ compared to IQF berries stored for 0 days. All data represent the mean of at least 3 independent experiments \pm SD. 

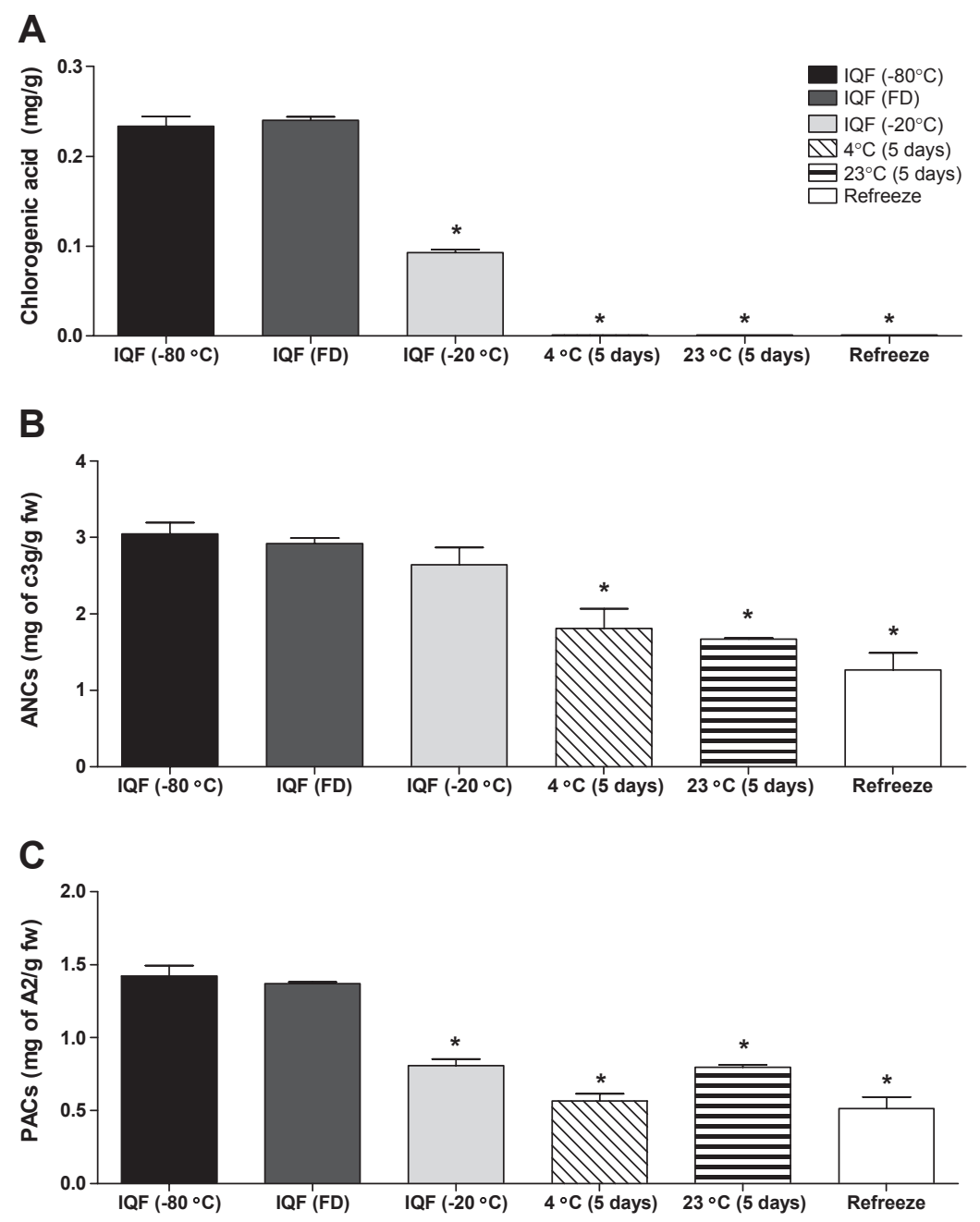

Fig. 2. Polyphenolic concentrations in individually quick-frozen wild blueberries. (A) Chlorogenic acid concentrations were determined using HPLC analyses of IQF WBB extracts post processing and storage. (B) Total anthocyanin concentration was measured by a pH differential method in extracts of IQF WBB that underwent specific processing and storage. (C) DMAC was used to quantify total proanthocyanidin concentration in IQF WBB subjected to postharvest processing and storage. All data represent the mean of at least 3 independent experiments \pm SD. *Represents $p<0.05$ compared with the control, IQF $\left(-80^{\circ} \mathrm{C}\right)$.

\subsection{Chlorogenic acid, total anthocyanins and total proanthocyanidins in IQF wild blueberries subjected to postharvest processing and storage}

CA concentrations were determined in IQF WBB after postharvest processing and storage (Fig. 2A). HPLC analyses reveal that IQF WBB stored at $-80^{\circ} \mathrm{C}$ or FD have comparable CA levels whereas those obtained from a supermarket source (subjected to transportation and held at $\left.-20^{\circ} \mathrm{C}\right)$ were significantly lower $(60.3 \%$ decrease). $\mathrm{CA}$ was not detected in IQF WBB stored at $4^{\circ} \mathrm{C}$ or $23^{\circ} \mathrm{C}$ for five days or in the IQF WBB that were subjected to refreezing.

Next, we determined total ANC concentration in postharvest processed and stored IQF WBB using the pH differential method (Fig. 2B). Despite our HPLC findings (Table 1) that revealed specific ANC concentrations in $\mathrm{IQF}\left(-20^{\circ} \mathrm{C}\right)$ to be significantly lower than those in $\mathrm{IQF}\left(-80^{\circ} \mathrm{C}\right)$, the overall total ANC concentration detected in 
Table 3

Anthocyanin concentrations in IQF wild blueberries after exposure to baking

\begin{tabular}{|c|c|c|c|c|c|c|c|}
\hline \multirow{3}{*}{ Peak } & \multicolumn{6}{|c|}{ Anthocyanins (mg/g) } & \\
\hline & \multirow{2}{*}{$\frac{\text { Control }}{0 \mathrm{~min}}$} & \multicolumn{3}{|c|}{$175^{\circ} \mathrm{C}\left(350^{\circ} \mathrm{F}\right)$} & \multicolumn{3}{|c|}{$220^{\circ} \mathrm{C}\left(425^{\circ} \mathrm{F}\right)$} \\
\hline & & $15 \mathrm{~min}$ & $30 \mathrm{~min}$ & $60 \mathrm{~min}$ & $15 \mathrm{~min}$ & $30 \mathrm{~min}$ & $60 \mathrm{~min}$ \\
\hline 1 & $0.245 \pm 0.036$ & $0.205 \pm 0.003$ & $0.162 \pm 0.013^{*}$ & nd & $0.204 \pm 0.011$ & $0.090 \pm 0.019 *$ & nd \\
\hline 2 & $0.262 \pm 0.007$ & $0.246 \pm 0.032$ & $0.178 \pm 0.011 *$ & nd & $0.236 \pm 0.030$ & $0.110 \pm 0.015^{*}$ & nd \\
\hline 3 & $0.120 \pm 0.018$ & $0.089 \pm 0.004^{*}$ & $0.078 \pm 0.003 *$ & nd & $0.099 \pm 0.011$ & $0.042 \pm 0.009 *$ & nd \\
\hline 4 & $0.161 \pm 0.025$ & $0.138 \pm 0.010$ & $0.101 \pm 0.013^{*}$ & nd & $0.133 \pm 0.001 *$ & $0.053 \pm 0.004^{*}$ & nd \\
\hline 5 & $0.121 \pm 0.005$ & $0.110 \pm 0.018$ & $0.081 \pm 0.007 *$ & nd & $0.117 \pm 0.019$ & $0.053 \pm 0.009 *$ & nd \\
\hline 6 & $0.143 \pm 0.013$ & $0.170 \pm 0.004$ & $0.136 \pm 0.004$ & nd & $0.177 \pm 0.016^{*}$ & $0.075 \pm 0.013^{*}$ & nd \\
\hline 7 & $0.230 \pm 0.001$ & $0.212 \pm 0.024$ & $0.156 \pm 0.007 *$ & nd & $0.215 \pm 0.033$ & $0.093 \pm 0.012^{*}$ & nd \\
\hline 8 & $0.046 \pm 0.005$ & $0.043 \pm 0.005$ & $0.030 \pm 0.001$ & nd & $0.044 \pm 0.006$ & $0.069 \pm 0.090$ & nd \\
\hline 9 & $0.094 \pm 0.009$ & $0.087 \pm 0.009$ & $0.062 \pm 0.002$ & nd & $0.091 \pm 0.012$ & $0.036 \pm 0.002 *$ & nd \\
\hline 10 & $0.325 \pm 0.012$ & $0.202 \pm 0.010^{*}$ & $0.153 \pm 0.031^{*}$ & nd & $0.207 \pm 0.022 *$ & $0.085 \pm 0.017 *$ & nd \\
\hline 11 & $0.333 \pm 0.007$ & $0.324 \pm 0.045$ & $0.235 \pm 0.015^{*}$ & nd & $0.339 \pm 0.065$ & $0.139 \pm 0.015^{*}$ & nd \\
\hline 12 & $0.138 \pm 0.013$ & $0.120 \pm 0.014$ & $0.060 \pm 0.053^{*}$ & nd & $0.118 \pm 0.012$ & $0.051 \pm 0.009^{*}$ & nd \\
\hline 13 & $0.091 \pm 0.010$ & $0.047 \pm 0.041$ & $0.024 \pm 0.043$ & nd & $0.074 \pm 0.008$ & $0.022 \pm 0.037 *$ & nd \\
\hline 14 & $0.042 \pm 0.001$ & $0.031 \pm 0.003$ & $0.029 \pm 0.006$ & nd & $0.038 \pm 0.009$ & $0.018 \pm 0.008 *$ & nd \\
\hline 15 & $0.087 \pm 0.014$ & $0.035 \pm 0.016^{*}$ & $0.031 \pm 0.008^{*}$ & nd & $0.038 \pm 0.008^{*}$ & $0.023 \pm 0.013^{*}$ & nd \\
\hline 16 & $0.060 \pm 0.006$ & $0.045 \pm 0.005$ & $0.039 \pm 0.014^{*}$ & nd & $0.048 \pm 0.004$ & $0.026 \pm 0.010^{*}$ & nd \\
\hline 17 & $0.162 \pm 0.020$ & $0.128 \pm 0.017$ & $0.109 \pm 0.040^{*}$ & nd & $0.139 \pm 0.020$ & $0.072 \pm 0.022^{*}$ & nd \\
\hline Total & $2.66 \pm 0.015$ & $2.233 \pm 0.020$ & $1.664 \pm 0.022$ & nd & $2.317 \pm 0.022$ & $1.056 \pm 0.026$ & $\mathrm{nd}$ \\
\hline
\end{tabular}

Retention of anthocyanins in individually quick-frozen wild blueberries post culinary preparations were measured using HPLC. Peak numbers correspond to individual anthocyanins denoted in Fig. 1 and 'nd' represents undetectable peaks. IQF $\mathrm{WBB}$ were baked at $175^{\circ} \mathrm{C}\left(350^{\circ} \mathrm{F}\right)$ or $220^{\circ} \mathrm{C}\left(425^{\circ} \mathrm{F}\right)$, extracted and analyzed. *Represents $p<0.05$, compared to control $(0 \mathrm{~min})$. All data represent the mean of at least 3 independent experiments $\pm \mathrm{SD}$.

IQF $\left(-20^{\circ} \mathrm{C}\right)$ using the $\mathrm{pH}$ differential method was not significantly different than control berries $\mathrm{IQF}\left(-80^{\circ} \mathrm{C}\right)$ or IQF FD berries. Additionally, ANC decreased by $40.6 \%, 45.1 \%$, and $58.5 \%$ in berries stored at $4{ }^{\circ} \mathrm{C}, 23^{\circ} \mathrm{C}$, and those subjected to refreezing, respectively.

Using the DMAC assay previously described, PAC concentrations were measured in postharvest processed or stored IQF WBB (Fig. 2C). Results revealed that freeze drying WBB does not influence PAC concentrations compared to control berries, whereas IQF $\left(-20^{\circ} \mathrm{C}\right) \mathrm{WBB}$ obtained from a distributor contained $43.1 \%$ less total PAC. Total PAC were decreased by $60.2 \%$ in berries stored at $4{ }^{\circ} \mathrm{C}$ for 5 days, $43.9 \%$ in those stored at $23^{\circ} \mathrm{C}$ for 5 days, and by $63.8 \%$ in berries subjected to refreeze conditions.

\subsection{Concentration of anthocyanins in IQF wild blueberries after culinary preparations}

Using HPLC methods, we determined ANC concentrations in IQF WBB that underwent three common culinary preparations including baking, boiling, and microwaving. Results indicated that baking IQF WBB at $175^{\circ} \mathrm{C}$ or $220^{\circ} \mathrm{C}$ for longer than 30 minutes significantly reduced ANC concentrations (Table 3). Furthermore, ANC were undetectable after baking for 60 minutes at either temperature. IQF WBB boiled for 1, 5, and 10 minutes retained 59.7\%, 76.2\%, and $85.5 \%$ less ANC, respectively than WBB that were never boiled (Table 4). Note that some of the loss of ANC in the boiled berries may be due to their release into the water as we know that ANC are water-soluble and we observed a color change in the boiling water. Lastly, IQF WBB exposed to microwaves for 1 minute showed minor changes in ANC concentrations where as microwave exposure for 3 and 5 minutes resulted in significant decreases $(28.0 \%$, and $68.1 \%$, respectively) compared to control berries (Table 4). 
Table 4

Anthocyanin concentrations in IQF wild blueberries after exposure to boiling and microwaving

\begin{tabular}{|c|c|c|c|c|c|c|c|}
\hline \multirow{3}{*}{ Peak } & \multicolumn{7}{|c|}{ Anthocyanins (mg/g) } \\
\hline & \multirow{2}{*}{$\begin{array}{l}\text { Control } \\
0 \mathrm{~min} \\
\end{array}$} & \multicolumn{3}{|c|}{ Boiled } & \multicolumn{3}{|c|}{ Microwaved } \\
\hline & & $1 \mathrm{~min}$ & $5 \mathrm{~min}$ & $10 \mathrm{~min}$ & $1 \mathrm{~min}$ & $3 \mathrm{~min}$ & $5 \mathrm{~min}$ \\
\hline 1 & $0.245 \pm 0.036$ & $0.106 \pm 0.006^{*}$ & $0.070 \pm 0.006^{*}$ & $0.035 \pm 0.007 *$ & $0.232 \pm 0.025$ & $0.193 \pm 0.028 *$ & $0.077 \pm 0.013^{*}$ \\
\hline 2 & $0.262 \pm 0.007$ & $0.112 \pm 0.011^{*}$ & $0.060 \pm 0.008 *$ & $0.044 \pm 0.007 *$ & $0.278 \pm 0.008$ & $0.204 \pm 0.020 *$ & $0.088 \pm 0.008^{*}$ \\
\hline 3 & $0.120 \pm 0.018$ & $0.049 \pm 0.002 *$ & $0.032 \pm 0.002 *$ & $0.016 \pm 0.005^{*}$ & $0.096 \pm 0.015$ & $0.088 \pm 0.019^{*}$ & $0.033 \pm 0.003 *$ \\
\hline 4 & $0.161 \pm 0.025$ & $0.069 \pm 0.002 *$ & $0.043 \pm 0.006^{*}$ & $0.025 \pm 0.005^{*}$ & $0.157 \pm 0.014$ & $0.124 \pm 0.012 *$ & $0.049 \pm 0.002 *$ \\
\hline 5 & $0.121 \pm 0.005$ & $0.053 \pm 0.007^{*}$ & $0.030 \pm 0.004^{*}$ & $0.019 \pm 0.006^{*}$ & $0.121 \pm 0.017$ & $0.090 \pm 0.003^{*}$ & $0.039 \pm 0.004^{*}$ \\
\hline 6 & $0.143 \pm 0.013$ & $0.056 \pm 0.005^{*}$ & $0.034 \pm 0.002 *$ & $0.019 \pm 0.005^{*}$ & $0.189 \pm 0.010^{*}$ & $0.159 \pm 0.027 *$ & $0.065 \pm 0.005^{*}$ \\
\hline 7 & $0.230 \pm 0.001$ & $0.115 \pm 0.006^{*}$ & $0.064 \pm 0.003^{*}$ & $0.042 \pm 0.010^{*}$ & $0.234 \pm 0.003$ & $0.179 \pm 0.001 *$ & $0.079 \pm 0.008^{*}$ \\
\hline 8 & $0.046 \pm 0.005$ & $0.006 \pm 0.007$ & $0.011 \pm 0.008$ & $0.004 \pm 0.003$ & $0.045 \pm 0.003$ & $0.035 \pm 0.004$ & $0.014 \pm 0.002$ \\
\hline 9 & $0.094 \pm 0.009$ & $0.028 \pm 0.001 *$ & $0.041 \pm 0.050^{*}$ & $0.009 \pm 0.003 *$ & $0.093 \pm 0.005$ & $0.072 \pm 0.008$ & $0.031 \pm 0.003^{*}$ \\
\hline 10 & $0.325 \pm 0.012$ & $0.133 \pm 0.006^{*}$ & $0.079 \pm 0.028 *$ & $0.044 \pm 0.012 *$ & $0.218 \pm 0.028 *$ & $0.186 \pm 0.038^{*}$ & $0.075 \pm 0.005^{*}$ \\
\hline 11 & $0.333 \pm 0.007$ & $0.126 \pm 0.014^{*}$ & $0.063 \pm 0.012^{*}$ & $0.048 \pm 0.012 *$ & $0.356 \pm 0.024$ & $0.267 \pm 0.021^{*}$ & $0.114 \pm 0.012^{*}$ \\
\hline 12 & $0.138 \pm 0.013$ & $0.053 \pm 0.003^{*}$ & $0.026 \pm 0.001^{*}$ & $0.018 \pm 0.007^{*}$ & $0.131 \pm 0.018$ & $0.109 \pm 0.018$ & $0.050 \pm 0.014^{*}$ \\
\hline 13 & $0.091 \pm 0.010$ & $0.036 \pm 0.011$ & $0.013 \pm 0.004 *$ & $0.014 \pm 0.001 *$ & $0.059 \pm 0.052$ & $0.03 \pm 0.027$ & $0.013 \pm 0.023^{*}$ \\
\hline 14 & $0.042 \pm 0.001$ & $0.018 \pm 0.004^{*}$ & $0.012 \pm 0.002 *$ & $0.008 \pm 0.003 *$ & $0.032 \pm 0.005$ & $0.021 \pm 0.004^{*}$ & $0.019 \pm 0.009 *$ \\
\hline 15 & $0.087 \pm 0.014$ & $0.019 \pm 0.005^{*}$ & $0.013 \pm 0.006^{*}$ & $0.007 \pm 0.001 *$ & $0.037 \pm 0.011^{*}$ & $0.025 \pm 0.002 *$ & $0.017 \pm 0.007^{*}$ \\
\hline 16 & $0.060 \pm 0.006$ & $0.024 \pm 0.007^{*}$ & $0.021 \pm 0.012^{*}$ & $0.007 \pm 0.001^{*}$ & $0.056 \pm 0.004$ & $0.032 \pm 0.011^{*}$ & $0.025 \pm 0.011^{*}$ \\
\hline 17 & $0.162 \pm 0.020$ & $0.068 \pm 0.017^{*}$ & $0.020 \pm 0.018^{*}$ & $0.027 \pm 0.002 *$ & $0.156 \pm 0.024$ & $0.100 \pm 0.012^{*}$ & $0.058 \pm 0.022^{*}$ \\
\hline Total & $2.66 \pm 0.015$ & $1.072 \pm 0.008$ & $0.632 \pm 0.016$ & $0.387 \pm 0.006$ & $2.491 \pm 0.020$ & $1.915 \pm 0.018$ & $0.848 \pm 0.011$ \\
\hline
\end{tabular}

IQF WBB were boiled or microwaved, extracted, and HPLC analyses were performed to determine anthocyanin retention. Peak numbers correspond to individual anthocyanins (Fig. 1). *Represents $p<0.05$, compared to control ( 0 min). All data represent the mean of at least 3 independent experiments $\pm \mathrm{SD}$.

\subsection{Chlorogenic acid, total anthocyanins and total proanthocyanidins in IQF wild blueberries post culinary preparation}

HPLC methods were used to determine CA concentrations in IQF WBB that were baked (Fig. 3A), boiled (Fig. 3B), or microwaved (Fig. 3C). CA levels decreased by $56.2 \%$ in WBB baked at $175^{\circ} \mathrm{C}$ for one hour and were not detectable after one hour of baking at $220^{\circ} \mathrm{C}$. Boiling diminished CA levels by $63.2 \%$ after $1 \mathrm{~min}, 95.2 \%$ after $5 \mathrm{~min}$, and $99.5 \%$ after $10 \mathrm{~min}$. Importantly, CA was detected in water samples that were collected after the boiling treatments were complete. CA concentration decreased in the berries and increased in the water samples over time. Conversely, microwaving IQF WBB up to 5 minutes did not significantly impact CA concentrations.

Decreased ANC ( $7.43 \%$ to $100 \%)$ were confirmed in the three culinary preparations using the pH differential method. Exceptions include total ANC in IQF WBB boiled for 5 minutes, which significantly deviated from the control by $14.7 \%$, and WBB that were microwaved for 1 min which increased by $12.9 \%$ compared to the control. In all culinary preparations PACs were reduced by $5 \%$ or more. Note that PACs, like CA, were detected in the recovered water samples.

\subsection{Effects of culinary preparation on the ability of WBB extracts to impact LPS-induced reactive oxygen species}

The ability of specific culinary preparations to influence the bioactive potential of IQF WBB was examined. As expected, LPS-induced ROS $(1.55 \pm 0.28, n=12)$ in SH-SY5Y cells was negated by incubation with GSH $(0.87 \pm 0.20, n=9)$ and furthermore by incubation with $25 \mu \mathrm{g} / \mathrm{ml}$ of crude blueberry extract (crude WBB, $1.05 \pm 0.15)$, to levels comparable to control cells $(1.00 \pm 0.11, n=12)$ (Fig. 4A). Exposure of IQF WBB to 




Total Anthocyanins



B

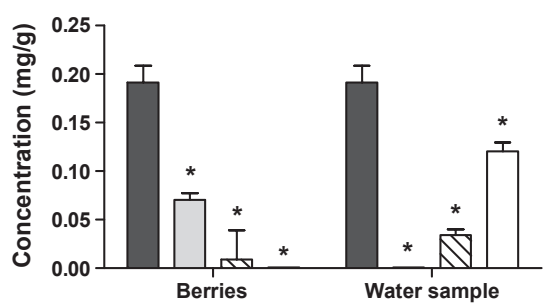

C

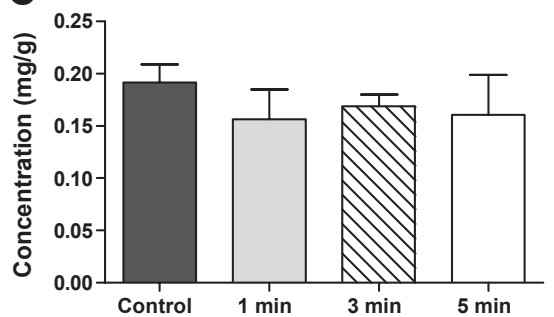

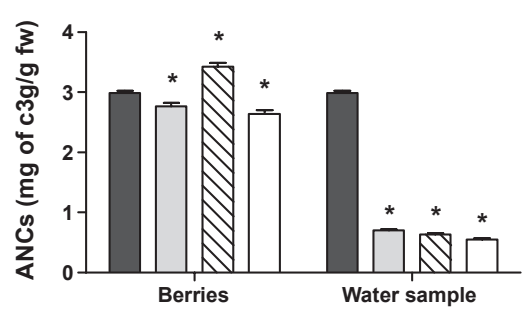

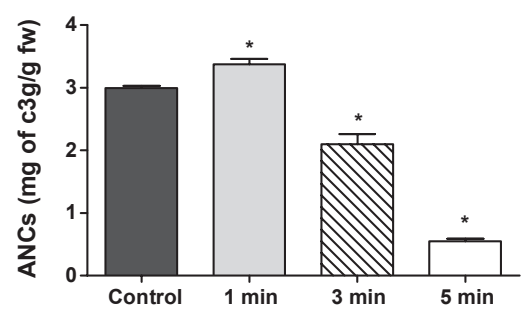

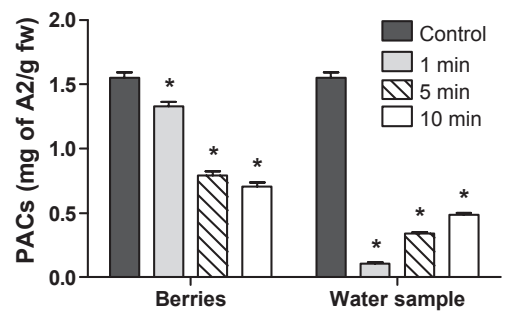

Total Proanthocyanins
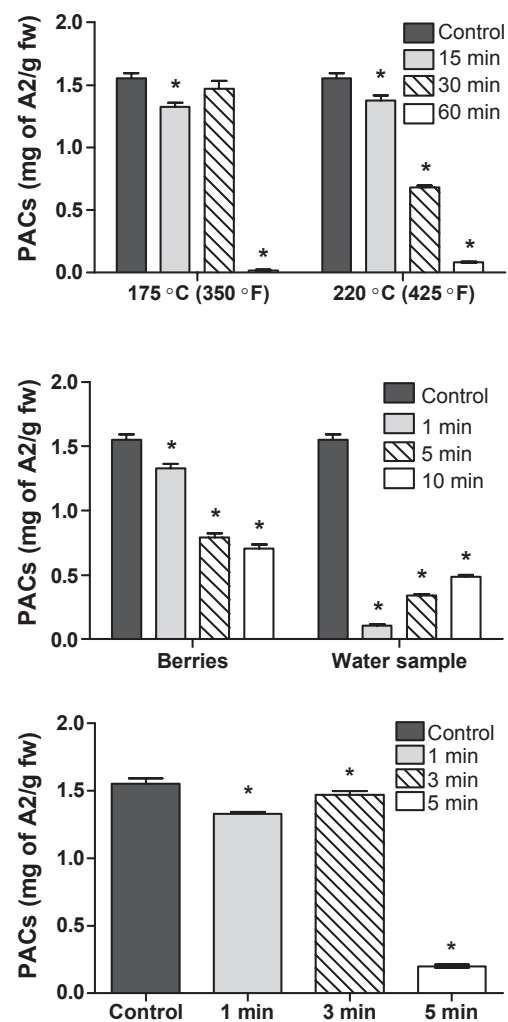

Fig. 3. Polyphenolic concentrations in individually quick-frozen wild blueberries post culinary preparations including (A) baking, (B) boiling, and (C) microwaving. Chlorogenic acid concentrations were determined using HPLC analyses on extracts of IQF WBB post culinary preparations (first column). Total anthocyanins (second column) were verified and total proanthocyanidins (third column) were determined for each culinary preparation using a $\mathrm{pH}$ differential method and DMAC assay, respectively. All data represent the mean of at least 3 independent experiments $\pm \mathrm{SD}$. *Represents $p<0.05$ compared with the control, IQF $\left(-80^{\circ} \mathrm{C}\right)$.

microwaves for 1 minute $(0.83 \pm 0.14, n=5)$ or 3 minutes $(0.90 \pm 0.07, n=5)$ did not impair the ability of WBB extract to decrease ROS accumulation in this model (Fig. 4B). Conversely, extracts of IQF WBB that had been exposed to microwaves for 5 minutes $(1.3 \pm 0.13, n=5)$ lost the capacity to decrease LPS-induced ROS (Fig. 4B). Lastly, IQF WBB extracts from baked (Fig. 4C) or boiled (Fig. 4D) WBB both demonstrated bioactivity by decreasing accumulation of LPS-induced ROS in SH-SY5Y cells.

\section{Discussion}

IQF WBB are available in the freezer section of many grocery markets and are also commonly used in processed foods available to consumers (9). The IQF process impedes enzymatic activity and prevents potential thermal degradation of polyphenolic compounds in WBB. For our studies we used IQF WBB fruit maintained at $-80^{\circ} \mathrm{C}$, freeze dried (FD), or distributed to industry sources and to grocery markets where they were stored at $-20^{\circ} \mathrm{C}$ prior to use or purchase. All WBB fruit used in the study was IQF within one day of harvest. We obtained our IQF $-20^{\circ} \mathrm{C}$ directly from a commercial distributor; these berries were stored primarily at $-20^{\circ} \mathrm{C}$ but we are unable to track the specific temperature variations that they may have encountered during the distributing process. 

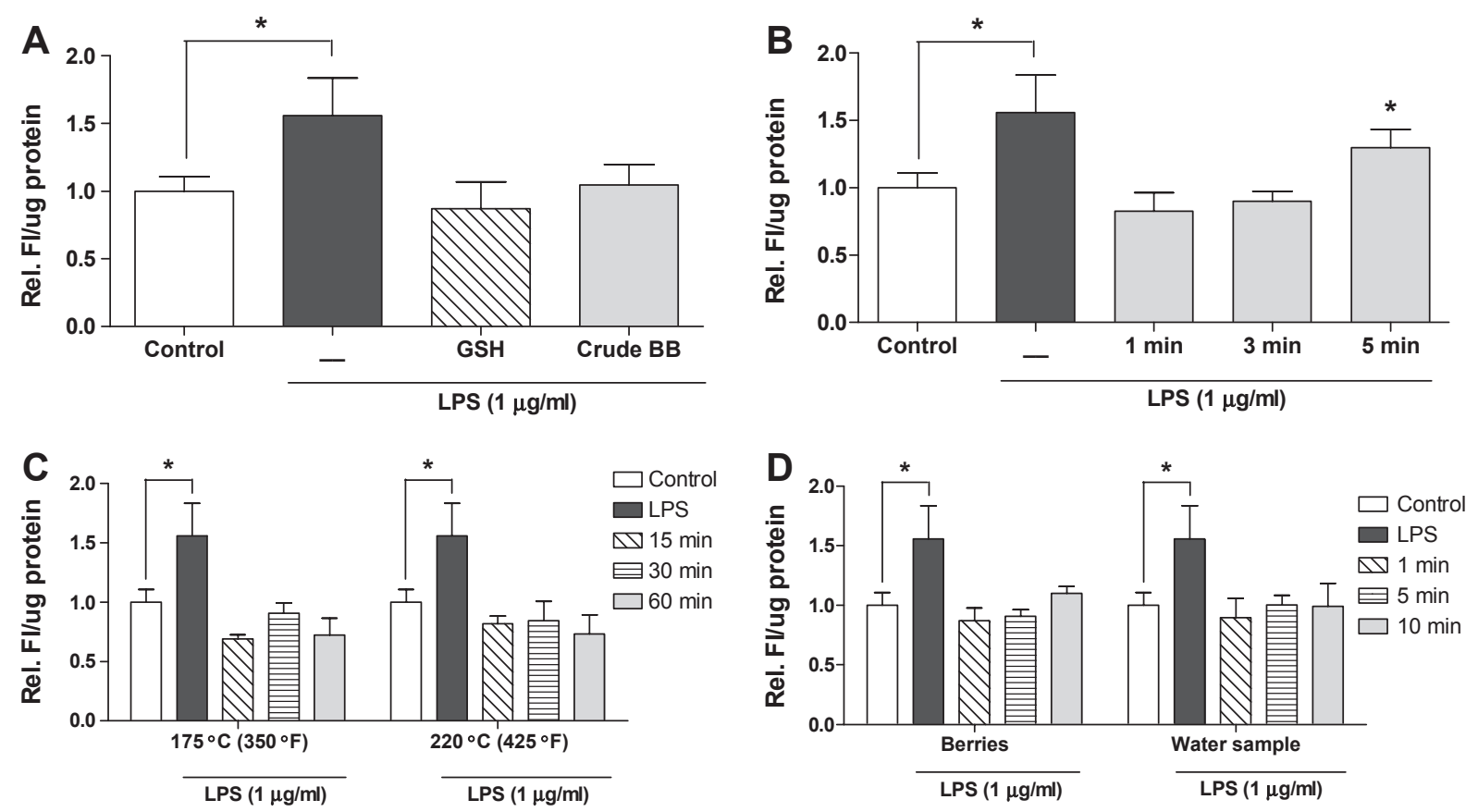

Fig. 4. The ability of blueberry extracts to reduce LPS-induced accumulation of ROS in SH-SY5Y cells. Cells were incubated with GSH (1 mM) or blueberry extract $(25 \mu \mathrm{g} / \mathrm{ml})$ in DCF-DA $(10 \mu \mathrm{M})$ prepared media, or DCF-DA media only (control, open bars), prior to LPS (1 $\mu \mathrm{g} / \mathrm{ml}$, filled bars) stimulation $(30 \mathrm{~min})$. All data represent the mean of at least 3 independent experiments \pm SD. $*$ Represents $p<0.05$ compared with the control.

Our results indicated that IQF $-80^{\circ} \mathrm{C}$ and IQF FD berries have similar ANC concentrations while the IQF $-20^{\circ} \mathrm{C}$ berries revealed significantly less ANC concentrations. These results were congruent with previous studies that indicated ANC degradation in fruit products to be temperature dependent and that storage conditions may influence losses in monomeric ANC $[14,17,18]$. The IQF $-80^{\circ} \mathrm{C}$ WBB obtained from WBANA are prepared as a composite from specific harvest locations across a region in which Vaccinium angustifolium grow wild; this composite ensures a homogenous sample for researchers interested in this species of blueberry fruit. The grocery market source of WBB used in this study are grown and harvested from the same geographical region as the WBANA WBB however we are unable to verify that the composite is identical to that of the composite obtained from WBANA. We suspect that our finding is due to prolonged storage at $-20^{\circ} \mathrm{C}$, temperature variations during the distributing process, or a combination of these factors.

Reduced levels of CA have been linked to the enzymatic activity of polyphenol oxidase (PPO) in fresh blueberry fruit and in other blueberry products $[15,19]$. Furthermore, the oxidation of CA has been associated with the degradation of ANC in blueberry fruit [19]. As expected, our results indicate that CA concentrations in IQF FD WBB did not deviate from IQF $-80^{\circ} \mathrm{C}$, whereas $\mathrm{CA}$ concentrations in $\mathrm{IQF}-20^{\circ} \mathrm{C}$ were significantly decreased. We hypothesize that the reduced levels of CA in IQF $-20^{\circ} \mathrm{C} \mathrm{WBB}$, and in the IQF WBB subjected to $4^{\circ} \mathrm{C}$ and $23^{\circ} \mathrm{C}$ storage conditions, are an indication of enzyme activity that occurred during temperature fluctuations or by oxidation of CA over time, respectively.

Potential changes in polyphenol content of commercial blueberry products after specific processing techniques may be of concern to consumers who desire bioactive compounds in their diets and for industry members who aspire to retain bioactive compounds in their products [20]. We investigated the effects of culinary preparations, relevant to the consumer and to the processors, on ANC, PAC, and CA concentrations in blueberry fruit. Our results demonstrate that baking, boiling and microwaving have the ability to decrease ANC and PAC concentrations in WBB over time; however, the total ANC in IQF WBB boiled for 5 minutes significantly deviated from the control by $14.7 \%$ and 
similarly, we observed a $12.9 \%$ increase of total ANC in IQF WBB that were microwaved for 1 min. Other reports of increased phytochemicals associated with culinary preparations include increased quercetin concentrations in onions (Allium cepa L.) exposed to sautéing, baking or boiling [21], and enhanced absorption of lycopene in tomatoes exposed to heat [22]. Moreover, a previous study suggests that hydrophilic antioxidant activity of wild blueberries is not negatively impacted by a variety of domestic cooking methods and is even increased in pan-fried frozen WBB [23]. Kalt et al. [24] determined that WBB products varied significantly in antioxidant capacity and that higher levels of ANC and total phenolics were present in WBB that were pureed at $60^{\circ} \mathrm{C}$ compared to those at $20^{\circ} \mathrm{C}$ [24]. It is suggested that this increase may be due to degradation of the WBB cell membranes which could allow the polyphenolic compounds to be more readily extracted from the tissue [23]. We suspect that the ANC increases we observed in this study are due to tissue alterations that enhanced ANC extraction, or a concentration effect due to moisture loss during the culinary treatment; however, further investigations are needed.

While total ANC and total PAC concentrations appear to decrease during the culinary preparations indicated in our study, CA concentrations in WBB that were baked or microwaved do not appear to be as strongly affected. Additionally, CA did decrease in boiled WBB, but was recovered in the water sample taken after the boiling treatment. CA concentrations may not have been as severely affected because the PPO enzyme, which acts upon CA, is not a heat-stable enzyme and it has been shown that short exposures to temperatures between $70-90^{\circ} \mathrm{C}$ can inactivate the enzyme [25]. This could potentially impede the oxidation and degradation of CA in our samples.

Overall, these findings caution consumers and processors alike that preparing IQF WBB using culinary techniques, especially those that employ heat for prolonged periods, may compromise the polyphenolic integrity of the final product. Importantly, we did detect CA, ANC, and PAC in water samples taken from the completed boiling treatments, which may be relevant to those who are concerned about the polyphenolic changes to berry fruits during jam preparation.

The impact that domestic cooking methods can have on fruits and vegetables is complex. Furthermore, these processes may initiate alterations to specific food components and thus influence the bioactive potential of foods subjected to culinary preparations. Strong evidence suggests that processing blueberries, and blueberry fruit products, results in decreased concentrations of ANC, PAC and CA. However, other studies imply that processing techniques may have little influence on oxygen radical absorbance capacity [16]. It has been established that $5 \mu \mathrm{g} / \mathrm{ml}$ of crude WBB extract significantly decreases the level of induced reactive oxygen species in SH-SY5Y cells [26]. Additionally, crude WBB extract does not compromise SH-SY5Y cell viability up to $75 \mu \mathrm{g} / \mathrm{ml}$ [27]. For our experiments, we assayed the bioactivity of crude extracts of WBB, post culinary preparations, in SH-SY5Y cells. Although ANC, PAC and CA concentrations were decreased by many of the culinary preparations investigated in this study, the ability of the WBB extract to diminish LPS-induced ROS in SH-SY5Y cells was not abolished, at least at $25 \mu \mathrm{g} / \mathrm{ml}$ concentrations. One exception was the $25 \mu \mathrm{g} / \mathrm{ml}$ of crude extract derived from WBB exposed to microwaves for 5 minutes, which did not maintain the ability to inhibit LPS-induced ROS. It is possible that a higher concentration of extract from WBB that were microwaved for 5 minutes may have a different effect in this inflammatory model and that the microwaving process over time could alter the berry tissue and in turn influence the extraction process that was performed for our experiments; however, a more involved study is needed to make this determination. In short, of all the culinary preparations that we examined, microwave exposure for 5 minutes had the most negative impact on bioactivity in this model system. With that, consumers or producers who desire to preserve the bioactivity of WBB may consider limiting microwave exposure of frozen blueberries.

\section{Summary}

This work characterized the retention of anthocyanins, proanthocyanidins and chlorogenic acid in wild blueberries, following various postharvest processing and storage conditions. We determined in this study that microwaves do not compromise levels of CA in WBB and that culinary preparations such as baking and boiling do not completely negate the bioactive potential of WBB to inhibit ROS accumulation in a neuronal model of inflammation (at $25 \mu \mathrm{g} / \mathrm{ml}$ blueberry extract). Furthermore, our results indicated that microwaving WBB for a 5 minute duration significantly decreased the berry's bioactive potential to inhibit ROS generation in SH-SY5Y cells. 


\section{Acknowledgments}

We would like to thank the Wild Blueberry Association of North America (WBANA) for providing the IQF WBB fruit and Future Ceuticals for providing the freeze-dried WBB material. We also thank both Aaron Massey and William Pressel for their assistance in the laboratory. Our gratitude is extended to Nicholas D. Gillitt, Ph.D., director of the Dole Nutrition Research Laboratory, for supplying us with the commercially available wild blueberries used in this study. This project was funded through USDA-ARS Project Nos. 0204-41510-001-24S and 58-6250-6-003. The contents of this publication do not necessarily reflect the views or policies of the US Department of Agriculture, nor does mention of trade names, commercial products, or organizations imply endorsement by the US Government.

\section{References}

[1] Joseph JA, Shukitt-Hale B, Willis LM. Grape juice, berries, and walnuts affect brain aging and behavior. J Nutr. 2009; 139: 1813S-7S.

[2] DeFuria J, Bennett G, Strissel KJ, Perfield JW 2nd, Milbury PE, Greenberg AS, et al. Dietary blueberry attenuates whole-body insulin resistance in high fat-fed mice by reducing adipocyte death and its inflammatory sequelae. J Nutr. 2009; 139: 1510-6.

[3] Stull AJ, Cash KC, Johnson WD, Champagne CM, Cefalu WT. Bioactives in blueberries improve insulin sensitivity in obese, insulin-resistant men and women. J Nutr. 2010; 140: 1764-8.

[4] Krikorian R, Shidler MD, Nash TA, Kalt W, Vinqvist-Tymchuk MR, Shukitt-Hale B, et al. Blueberry supplementation improves memory in older adults. J Agric Food Chem. 2010; 58: 3996-4000.

[5] Yi W, Fischer J, Krewer G, Akoh CC. Phenolic compounds from blueberries can inhibit colon cancer cell proliferation and induce apoptosis. J Agric Food Chem. 2005; 53: 7320-9.

[6] US Highbush Blueberry Council [website]. Blueberry new products continue at rapid pace. (2010). Available from: http://www.blueberry.org/news/news-2010/news-2010.html

[7] North American Blueberry Council [website]. Blueberry per capita consumption on the rise. (2010). Available from: http://www.blueberry.org/news/news-2010/news-2010.html

[8] Borges G, Degeneve A, Mullen W, Crozier A. Identification of flavonoid and phenolic antioxidants in black currants, blueberries, raspberries, red currants and cranberries. J Agric Food Chem. 2010; 58: 3901-9.

[9] Kalt W, Ryan DA, Duy JC, Prior RL, Ehlenfeldt MK, Vander Kloet SP. Interspecific variation in anthocyanins, phenolics, and antioxidant capacity among genotypes of highbush and lowbush blueberries (Vaccinium section cyanococcus spp.). J Agric Food Chem. 2001; 49: 4761-7.

[10] Schreckinger ME, Wang J, Yousef GG, Lila MA, De Mejia EG. Antioxidant capacity and in vitro inhibition of adipogenesis and inflammation by phenolic extracts of Vaccinium floribundum and Aristotelia chilensis. J Agric Food Chem. 2010; 58: 8966-76.

[11] Grace MH, Ribnicky DM, Kuhn P, Poulev A, Logendra S, Yousef GG, et al. Hypoglycemic activity of a novel anthocyanin-rich formulation from lowbush blueberry, Vaccinium angustifolium Aiton. Phytomedicine. 2009; 16: 406-15.

[12] Cheng GW, Breen PJ. Activity of phenylalanine ammonia-lyase (PAL) and concentrations of anthocyanin and phenolics in developing strawberry fruit. J Am Soc Hortic Sci. 1991; 116: 865-9.

[13] Prior RL, Fan E, Ji H, Howell A, Nio C, Payne MJ, et al. Multi-laboratory validation of a standard method for quantifying proanthocyanidins in cranberry powders. J Sci Food Agric. 2010; 190: 1473-8.

[14] Wang W-D, Xu S-Y. Degradation kinetics of anthocyanins in blackberry juice and concentrate. JFood Eng. 2007; 82: 271-5.

[15] Kader F, Irmouli M, Nicolas JP, Metche M. Involvement of blueberry peroxidase in the mechanisms of anthocyanin degradation in blueberry juice. Food Chem Toxicol. 2002; 67: 910-5.

[16] Howard LR, Castrodale C, Brownmiller C, Mauromoustakos A. Jam processing and storage effects on blueberry polyphenolics and antioxidant capacity. J Agric Food Chem. 2010; 58: 4022-9.

[17] Mishra DK, Dolan KD, Yang L. Confidence intervals for modeling anthocyanin retention in grape pomace during nonisothermal heating. J Food Sci. 2008; 73: E9-15.

[18] Hager TJ, Howard LR, Prior RL. Processing and storage effects on monomeric anthocyanins, percent polymeric color, and antioxidant capacity of processed blackberry products. J Agric Food Chem. 2008; 56: 689-95.

[19] Kader F, Rovel B, Girardin M, Metche M. Mechanism of browning in fresh highbush blueberry fruit (Vaccinium corymbosum L). Role of blueberry polyphenol oxidase, chlorogenic acid and anthocyanins. J Sci Food Agric. 1997; 74: 31-4.

[20] Brownmiller C, Howard LR, Prior RL. Processing and storage effects on monomeric anthocyanins, percent polymeric color and antioxidant capacity of processed blueberry products. J Food Sci. 2008; 73: H72-9.

[21] Amarowicz R, Carle R, Dongowski G, Durazzo A, Galensa R, Kammerer D, et al. Influence of postharvest processing and storage on the content of phenolic acids and flavonoids in foods. Mol Nutr Food Res. 2009; 53(Suppl 2): S151-83.

[22] Unlu NZ, Bohn T, Francis DM, Nagaraja HN, Clinton SK, Schwartz SJ. Lycopene from heat-induced cis-isomer-rich tomato sauce is more bioavailable than from all-trans-rich tomato sauce in human subjects. Br J Nutr. 2007; 98: 140-6. 
[23] Murphy RR, Renfroe MH, Brevard PB, Lee RE, Gloeckner JW. Cooking does not decrease hydrophilic antioxidant capacity of wild blueberries. Int J Food Sci Nutr. 2009; 60(Suppl 2): 88-98.

[24] Kalt W, McDonald JE, Donner H. Anthocyanins, phenolics, and antioxidant capacity of processed lowbush blueberry products. Food Chem Toxicol. 2000; 65: 390-3.

[25] Vamos-Vigyazo L. Polyphenol oxidase and peroxidase in fruits and vegetables. Crit Rev Food Sci Nutr. 1981 ; $15: 49-127$.

[26] Gustafson S, Dunlap K, McGill C, Kuhn T. A nonpolar blueberry fraction blunts NADPH oxidase activation in neuronal cells exposed to tumor necrosis factor alpha. Oxid Med Cell Longev. 2012: 1-12. doi: 10.1155/2012/768101 (open access article).

[27] Gustafson S, Barth B, McGill C, Clausen T, Kuhn T. Alaska wild blueberry extracts inhibit a magnesium-dependent neutral sphingomyeliase activity in neurons exposed to TNF. Curr Top Nutraceut R. 2007; 5: 183-8. 